\title{
Streptococcus agalactiae infection kills red tilapia with chronic Francisella noatunensis infection more rapidly than the fish without the infection
}

\author{
Wanna Sirimanapong ${ }^{\mathrm{a}^{*}}$, Kim D. Thompson ${ }^{\mathrm{b}}$, Andrew P. Shinn ${ }^{\mathrm{c}}$, Alexandra Adams ${ }^{\mathrm{d}}$ and \\ Boonsirm Withyachumnarnkul ${ }^{\mathrm{e}}$
}

${ }^{\text {a}}$ Veterinary Aquatic Animal Research Health Care Unit, Department of Clinical Sciences and Public Health, Faculty of Veterinary Science, Mahidol University, Nakhon-pathom 73170, Thailand

${ }^{\mathrm{b}}$ Moredun Research Institute, Pentlands Science Park, Bush Loan, Penicuik, Scotland, UK ${ }^{c}$ Fish Vet Group Asia Limited, 21/359 Premjairard Road, Saensook, Muang Chonburi, Chonburi, 20130, Thailand

${ }^{\mathrm{d}}$ Institute of Aquaculture, Faculty of Natural Sciences, University of Stirling, Stirling, Scotland, UK

eAquaAcademy Farm, Tha Chana, Surat Thani 84170; and Department of Anatomy, Faculty of Science, Prince of Songkla University, Hatyai, Songkla 90112, Thailand

\footnotetext{
Accepted refereed manuscript of: Sirimanapong W, Thompson KD, Shinn AP, Adams A \& Withyachumnarnkul B (2018) Streptococcus agalactiae infection kills red tilapia with chronic Francisella noatunensis infection more rapidly than the fish without the infection. Fish and Shellfish Immunology, 81, pp. 221-232.

DOI: https://doi.org/10.1016/j.fsi.2018.07.022

(C) 2018, Elsevier. Licensed under the Creative Commons Attribution-NonCommercial-NoDerivatives 4.0 International http://creativecommons.org/licenses/by-nc-nd/4.0/
}

*Corresponding author: Wanna Sirimanapong

Tel.: +662-441-5242 ext. 1520; Fax: +662-441-0773

E-mail:wanna.sir@mahidol.ac.th 


\section{Abstract}

In this study we examined the effect that a Francisella noatunensis (Fno) infection had on hybrid red tilapia (Oreochromis niloticus $\times$ Oreochromis mossambicus) subsquently infected with Streptococcus agalactiae. A variety of hemato-immunological parameters (haematocrit, total red blood cell count, mean corpuscular volume, total white blood and differential cell counts, total plasma protein, plasma lysozyme and plasma peroxidase activities, and respiratory burst and phagocytic activities of head-kidney macrophages) were measured in hybrid red tilapia that had been previously exposed to an Fno outbreak in a tilapia grow-out farm. The head-kidneys of these apparently healthy survivors, when checked by PCR were found to be Fno-positive with hemato-immunological parameters that were similar to fish without an a priori infection. The only exception was the percentage lymphocyte count in the peripheral blood, which was slightly, but significantly, lower in the Fno-infected fish, compared to those without the infection. When experimentally infected with S. agalactiae, the Fno-infected fish died more rapidly and at a significantly higher rate than fish without the infection. During the challenge, the hemato-immunological parameters of both groups of fish were very similar, although the Fno-infected fish, challanged with $S$. agalactiae expressed significantly higher plasma lysozyme and peroxidase activities, and their head kidney macrophages had significantly higher respiratory burst activity compared to non-Fno-infected fish challanged with S. agalactiae. The only two parameters for which Fno-infected fish showed significantly lower expressions than that of their non-infected counterparts were haematocrit and total red blood cell count. The cause of the rapidity and higher rates of mortality observed in the Fno-infected fish when challenged with S. agalactiae is unknown; but it may be due to a reduced erythropoiesis capability within the head-kidney because of the presence of Fno.

Keywords: Francisella noatunensis subsp. orientalis; Streptococcus agalactiae; red tilapia; francisellosis; streptococcosis; sequential infections; bacterial pathogens 


\section{Introduction}

Nile tilapia (Oreochromis niloticus) and hybrid red tilapia (Oreochromis niloticus $\times$ Oreochromis mossambicus) are important aquaculture species that are farmed globally. In many locations where they are farmed, they are naturally infected by the bacterium Francisella noatunensis subsp. orientalis (Fno) (previously known as F. asiatica), resulting in the disease francisellosis, with associated mass mortalities [1-5]. This bacterium is a Gramnegative, pleomorphic, coccobacillus, which is a facultative intracellular pathogen able to infect many fish species, including tilapia. The predominant gross pathology in Fno-infected fish is the presence of multiple granulomas on the surface of the kidney, spleen and liver [6, 7]. Infection in the anterior part of the kidney, or head-kidney, may result in a decrease in the immunocompetence of the fish, since this organ is the site of erythropoiesis, myelopoiesis and lymphopoiesis, where several immunocompetent cells are generated [8]. Fish can also be infected by Fno without any clinical manifestation of the disease $[9,10]$. From a recent study conducted in Thailand, about $60 \%$ of the farmed red tilapia were found to be sub-clinically infected with Fno, which has been shown to be vertically transmitted [10].

One of the most common pathogens associated with mass mortality of farmed tilapia is the bacterium Streptococcus agalactiae, which infects both Nile and red tilapia [11]. This bacterium has also been reported to cause subclinical infections in tilapia [12, 13]. Moreover, natural co-infection of $S$. agalactiae and Fno, have been reported in farmed $O$. niloticus resulting in mass mortalities [10]. The site of infection of $S$. agalactiae is the liver, spleen, heart, kidney and brain, but mortality is usually associated with the infection of the brain [11], while the site of infection of Fno is principally within the spleen and head-kidney [14]. It is possible that an Fno infection in the head-kidney of tilapia might affect the fish's haematology and immune system, thus reducing its ability to effectively protect itself against attack by opportunistic pathogens. 
In the current study, red tilapia surviving a natural Fno infection, and shown to be sub-clinically infected with the bacterium in their head-kidney, were subsequently experimentally infected with $S$. agalactiae. Haematological and innate immune responses were measured in Fno-infected and non-infected fish, before and after challenge with $S$. agalactiae, as well as monitoring the survival rate of the infected fish.

\section{Materials and methods}

\subsection{Experimental animals}

Three hundred hybrid red tilapia (Oreochromis niloticus $\times$ Oreochromis mossambicus), 200-300 g in weight, which had survived a natural Fno infection were collected from a commercial farm site in Nakhornsawan Province, Thailand. The infection, which resulted in approximately $45 \%$ mortality, occurred a few months before sample collection, by which time the mortalities had completely stopped. Ten of these fish were randomly collected and their head-kidneys screened for the presence of residual Fno by polymerase chain reaction (PCR), targeting the $16 \mathrm{~S}$ rRNA sequence (F11, 5'-TAC CAG TTG GAA ACG ACT GT-3' and F5, 5'-CCT TTT TGA GTT TCG CTCC-3') [1]; all fish were found to be positive for the bacterium. No $S$. agalactiae infection was detected by PCR using the specific primers (F5'-CGCT $\neg$ GAGGTTTGGTGTTTACA-3' and R5'-CACTCCTACCAACGTTCTTC-3') [ 15] . This group of red tilapia was, therefore, considered to be sub-clinically infected with Fno. Three hundred control hybrid red tilapia of the same size were collected from another farm in the same Province that had no previous history of any disease outbreak, including infection with Fno. Ten of these fish were also randomly sampled and their kidneys screened for presence of Fno by PCR, all of which were found to be Fno-negative. The fish were brought to the wet lab at Mahidol University, Bangkok, and stocked in 350-L fibreglass tanks, with 30 individuals per tank; each tank was provided with a separate water supply and drainage, using a flow-through system. 
Each of the two groups were then further divided into a group to be experimentally infected with $S$. agalactiae and another that would not be infected with the pathogen, resulting in four groups altogether: G1, Fno-free fish without S. agalactiae challenge; G2, Fno-infected fish without S. agalactiae challenge; G3, Fno-free fish with S. agalactiae challenge; and G4, Fno-infected fish with S. agalactiae challenge. Five tanks were used for each group with 30 fish per tank (150 fish per group). Water quality was monitored daily to ensure optimal water conditions for the fish, with the following water quality values maintained: dissolved oxygen, $>4 \mathrm{ppm}$; total ammonia nitrogen, $<0.5 \mathrm{ppm}$; total nitrite, $<0.5$ ppm; $\mathrm{pH}, 7.0-7.5$ and water temperature, $28 \pm 1{ }^{\circ} \mathrm{C}$. A water exchange of $20-30 \%$ was performed daily.

\subsection{Haematological and immunological parameters}

One fish was randomly sampled from each tank (5 fish per group) to assess their haemato-immunological parameters at 0 hour post-infection (hpi), before performing the $S$. agalactiae challenge; thus, the fish tested were 10 Fno-free control fish (from G1 and G3 combined) and 10 Fno-infected fish (from G2 and G4 combined). The parameters measured included haematological parameters: haematocrit (Hct), total red blood cell (RBC) count, mean corpuscular volume (MCV), total white blood cell (WBC) and differential WBC count; plasma-related parameters (lysozyme activity, peroxidase activity and total protein levels); and head-kidney macrophage activity [respiratory burst (RBA) and phagocytic activities].

\subsubsection{Haematology}

Blood samples were collected from the caudal vein of the fish and all the parameters described determined using the protocols outlined by Sirimanapong et al. [16]. Briefly, $1 \mathrm{~mL}$ of blood was withdrawn from the tail vein of individual fish and immediately mixed with heparin $(200 \mathrm{U} / \mathrm{mL})$. The withdrawn blood, $100 \mu \mathrm{L}$, was immediately used to determine Hct, 
total RBC count, total and differential WBC counts and MCV. The rest of the blood was centrifuged at 3,000× $\mathrm{g}$ for $5 \mathrm{~min}$, plasma collected, and kept at $-70{ }^{\circ} \mathrm{C}$ for further analysis.

The Hct values were determined by placing well-mixed blood into haematocrit capillary tubes, which were centrifuged at 3,000 $\times \mathrm{g}$ for $5 \mathrm{~min}$; and the values calculated as percentage of packed RBC volume against the whole blood volume. For total RBC and WBC counts, blood $(20 \mu \mathrm{L})$ was added to $4 \mathrm{~mL}$ Natt-Herricks's solution and mixed thoroughly before counting on a Neubauer chamber. From the values of Hct and total RBC count, MCV was determined according to the formula: $\mathrm{MCV}(\mathrm{fL})=(\mathrm{Hct} \times 10) /$ total $\mathrm{RBC}\left(\times 10^{6}\right.$ cells $\left./ \mu \mathrm{L}\right)$. WBC differential count was carried out by preparing a smear of whole blood on microscope slides, stained by Wright-Giemsa and examined under light microscopy and the percentage of neutrophils, lymphocytes, monocytes, eosinophils, basophils and thrombocytes determined. At least 200 cells were counted for each differential count.

\subsubsection{Parameters in plasma}

The plasma was thawed and lysozyme activity, plasma peroxidase activity and total protein content determined. Lysozyme activity was determined according to the method previously described by Morgan et al. [17]. Briefly, a suspension of $0.2 \%$ (w/v) Micrococcus lysodeikticus acquired from Sigma, UK was prepared in $0.04 \mathrm{M}$ phosphate buffer saline (PBS) and $190 \mu \mathrm{L}$ of the bacterial mixture was placed into microtitre plate wells, and control wells contained only the buffer. Plasma samples $(10 \mu \mathrm{L})$ were added into the wells to make a final volume of $200 \mu \mathrm{L}$ well ${ }^{-1}$. Each plasma sample tested consisted of four replicates. The wells were incubated at room temperature $\left(32 \pm 1^{\circ} \mathrm{C}\right)$ and the absorbency at $540 \mathrm{~nm}$ read at 1 and 5 min after the incubation. One unit of lysozyme activity was defined as the amount of sample causing a decrease in absorbance at $0.001 / \mathrm{min}$.

Plasma peroxidase was determined using a modification of the method outlined by Quade and Roth [18] and Sitjà-Bobadilla et al. [19]. The plasma sample (15 $\mu \mathrm{L})$ and Hanks' 
balanced salt solution without $\mathrm{Ca}^{2+}$ and $\mathrm{Mg}^{2+}(135 \mu \mathrm{L})$ were added to the flat bottomed well

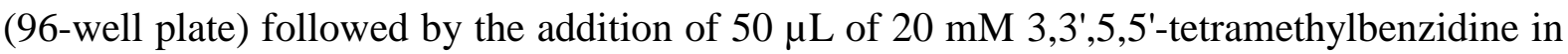
substrate buffer (40 $\mathrm{mM}$ acetic acid) containing $0.1 \% \mathrm{H}_{2} \mathrm{O}_{2}$. The reaction was stopped with $50 \mu \mathrm{L}$ of $2 \mathrm{M} \mathrm{H}_{2} \mathrm{SO}_{4}$ and read with an ELISA reader at $450 \mathrm{~nm}$.

Total plasma protein was analysed using a Bradford assay. A protein standard series (from 0 to $2 \mathrm{mg} / \mathrm{mL}$ ) were prepared by diluting $6 \mathrm{mg}$ bovine serum albumin in PBS. Twenty $\mu \mathrm{L}$ of sample or standard was added to the wells of a 96 well plate, to which $200 \mu \mathrm{L}$ of Bradford solution (Sigma, UK) was then added and mixed thoroughly on a plate shaker for $30 \mathrm{sec}$. After $5 \mathrm{~min}$, the plates were read at an absorbance of $595 \mathrm{~nm}$ on a microplate reader. A standard curve of protein concentration against absorbance at $595 \mathrm{~nm}$ was plotted and the level of total protein in the samples determined.

\subsubsection{Head-kidney macrophage activity}

Samples of head-kidney were collected to examine head-kidney macrophage activity, i.e. RBA with and without stimulation by phorbol myristate acetate (PMA), reactive oxygen species (ROS) stimulation index and phagocytosis, all of which were performed as previously described by Sirimanapong et al. [20]. To determine the RBA, head-kidney phagocytes were isolated and the cell suspension was placed into a 96 well tissue culture plate $(100 \mu \mathrm{L} / \mathrm{well})$, 10 wells per fish, and incubated at $28-30{ }^{\circ} \mathrm{C}$ for $2 \mathrm{~h}$. The absorbance of the wells was measured at $610 \mathrm{~nm}$ using a micro-plate reader (Synergy HT; Bio Tek Instruments, Winooski, VT, USA) and the results expressed as an absorbance at $610 \mathrm{~nm}$ per $10^{6}$ cells. The ROS stimulation index was calculated from the equation PMA-stimulated ROS/mean unstimulated ROS.

Phagocytic activity by head-kidney macrophages was determined by placing $100 \mu \mathrm{L}$ of the head-kidney cell suspension onto microscopic slides, which were then placed in a moist incubation chamber for $1 \mathrm{~h}$ at room temperature $\left(32 \pm 1^{\circ} \mathrm{C}\right)$ to allow adherence of cells 
to the slide. Yeast, Saccharomyces cerevisiae, $10^{7}$ yeast cells/mL in L-15 medium (Sigma, UK), was used as the phagocytic particles and $100 \mu \mathrm{L}$ of the yeast suspension was placed into one circle of attached cells and $100 \mu \mathrm{L}$ of the medium, as control, into the second circle. The slides were incubated for $1 \mathrm{~h}$ at room temperature $\left(32 \pm 1^{\circ} \mathrm{C}\right)$ to allow phagocytosis to take place and then washed with L-15 medium. The cells were fixed with $100 \%$ methanol (100 $\mu \mathrm{L}$ ) for $5 \mathrm{~min}$ and stained with Giemsa stain, and the number of phagocytes containing yeast was counted for a total 200 phagocytes. The phagocytic index (PI), phagocytic activity (PA), and phagocytic capacity (PC) were determined as described previously; PC1, PC3, PC5 and $\mathrm{PC} \geq 6$ signify the percentage of phogocytes containing 1 or more yeast cells [21].

\subsection{Streptococcus agalactiae challenge}

A virulent strain of S. agalactiae serotype Ia (MUVS2017SA02), isolated from a field outbreak, was cultured in tryptone soy agar (TSA, Oxoid England) for $24 \mathrm{~h}$. Three to five colonies of the bacteria were randomly selected from the plate and placed into $100 \mathrm{~mL}$ of sterile tryptic soy broth (TSB). The bacteria were cultured to late logarithmic growth in a shaking incubator $\left(150 \mathrm{rpm}, 28^{\circ} \mathrm{C}\right)$ (Incu-shakerTM 10L, Benchmark, NJ) for $24 \mathrm{~h}$ and the concentration of the bacterial suspension determined using spectrophotometer at $\mathrm{OD}_{600} \mathrm{~nm}$ and the concentration confirmed with a plate counting method as colony forming units.

The fish in G1 and G3 were individually injected with $0.1 \mathrm{~mL}$ of TSB, while those in G2 and G4 were individually injected intraperitoneally with $0.1 \mathrm{~mL}$ TSB containing $2.4 \times 10^{7}$ cfu/mL S. agalactiae. At 3, 6, 12, 24 and 48 h post-injection, one fish per tank (thus, five fish per group at each time-point) was randomly sampled and determined for haematoimmunological parameters as described. Mortality was monitored twice daily for 2 weeks in seperate tanks using 24 fish per tank in five replicate tanks per group. Moribund fish were clinically assessed and tissue swabs from kidney were cultured onto TSA and the presence of S. agalactiae in the cultures were confirmed by PCR assay [15]. 


\subsection{Statistical analysis}

All data were analysed using one-way analysis of variance (ANOVA), general linear models, and pairwise comparison (Tukey) of the means. All statistical tests were performed using SPSS software (Version 19) under license to Mahidol University. The differences were considered statistically significant when $p<0.05$. Principal Components Analyses (PCAs) were conducted within Statistica 10 (StatSoft, USA) on the raw data obtained for 24 measured immunological parameters. An ANOVA with a post-hoc Tukey HSD test with both Bonferroni and Holm's correction were ran on the factor scores for the first principal component derived from each analysis. Both tests were applied to verify that the derived $p$ values were genuine and controlled for Type 1 errors (i.e. false positives). Statistically significant differences were accepted at $p<0.05$.

\subsection{Ethics statement}

This study was approved by the Faculty of Veterinary Science-Animal Care and Use Committee, Mahidol University (FVS-ACUC; permit number MUVS-2016-05-21).

\section{Results}

\subsection{Haemato-immunological parameters of Fno-free and Fno-infected red tilapia}

Before performing the $S$. agalactiae challenge, the haemato-immunological parameters of the Fno-free and Fno-infected fish were compared and most of the parameters tested were not statistically different between the two groups (Table 1). The only parameter that was statistically different was the percentage of lymphocytes, which was significantly lower $(p<0.05)$ in the Fno-infected fish compared to the Fno-free fish (i.e. approx. $85 \%$ of those compared to the Fno-free fish). 


\subsection{Survival of $\boldsymbol{F n o}$-free and $\boldsymbol{F n o}$-infected red tilapia following $S$. agalactiae challenge}

The survival of the fish following the S. agalactiae challenge is presented in Figure 1, which shows that the G4 fish died rapidly with $<10 \%$ survival by at the second day postinfection (dpi), while the survival of the fish in G3 at the same time point was $>10 \%$. From 11 to 14 dpi, the survival of G4 was $3.3( \pm 3.5) \%$, while that of G3 was $46.7( \pm 5.4) \%$. The moribund fish had an erratic swimming pattern, exophthalmos and skin lesions. Swabs taken from the head-kidney, liver and spleen, streaked on agar produced colonies of bacteria which were characterised as $S$. agalactiae from their biochemical profile. These tests included oxidase, catalase, methyl red Voges-Proskauer, bile-aesculin tolerance, hippurate hydrolysis, fermentation of sorbitol, pyrrolidonylarylamidase, arginine deamination and leucine aminopeptidase [22]. The identity of the bacteria was confirmed by PCR (see section 2.1).

\subsection{Hemato-immunological parameters of the Fno-free and Fno-infected red tilapia during $S$. agalactiae challenge}

Following the $S$. agalactiae challenge, several changes in the measured parameters were seen. Although a comparison was made between the four groups at each time-point, the difference between G3 vs. G4 was interesting since it revealed how the Fno-infected fish responded differently to the challenge to that of the Fno-free fish.

\subsubsection{Haematology}

Following the S. agalactiae infection, the Hct of G3 and G4 fish were generally lower than those of their respective controls, G1 and G2, with statistically significant differences obtained at several time-points: G1 vs. G3, at 3, 12 and 48 hpi; G2 vs. G4, at 12 and 48 hpi

(Fig. 2A). Comparing between G3 vs. G4, the Hct of G4 fish was significantly lower than 
that of G3 fish at 12 and 48 hpi, suggesting that the Fno-infected fish had become more anaemic than the Fno-free fish following infection with $S$. agalactiae.

The RBC value of G3 fish was significantly higher than that of G1 fish at $24 \mathrm{hpi}$, while the RBC values of G4 fish was significantly lower than that of G2 fish at 12 hpi. Comparing between G3 vs. G4 fish, the RBC count of G4 was significantly lower than that of G3 at 12 and 24 hpi (Fig. 2B). Therefore, S. agalactiae infection in Fno-free fish had either "no-effect" or resulted in a higher RBC count, while the infection in the Fno-infected fish had either "no-effect" or resulted in a lowering of the RBC count.

The values of MCV obtained for G3 fish was significantly lower than that of G1 fish at 12 and 24 hpi, while corresponding values in G4 fish did not differ to those of G2 fish at any time-point. No significant differences between G3 and G4 fish were detected at any timepoint (Fig. 2C). Therefore, compared to the non-challenge fish, infection with $S$. agalactiae caused a decrease in the MCV value in the Fno-free fish, but not in the Fno-infected fish.

The total WBC count, and the differential count for lymphocytes and thrombocytes for the four groups of fish are presented in Figure 3. No differences were found in the percentage of neutrophils, eosinophils and monocytes between the groups of fish, and therefore the data for these are not presented in the figure (see Supplemental Table 1). The total WBC count of G3 fish was significantly higher than that of G1 fish at 6, 12, 24 and 48 hpi, whereas the WBC count of G4 fish was only significantly higher than that of G2 fish at 48 hpi. Comparing G3 vs. G4 fish, the latter had significantly lower levels of WBC than G3 fish at 12 hpi. The results, therefore, suggested that there is an increase in the total WBC count in both Fno-free and Fno-infected fish in response to the S. agalactiae infection, but this response was statistically higher in Fno-free fish, compared to the Fno-infected fish, at certain points during the challenge.

When comparing the percentage of lymphocytes in G1 vs. G3 fish, the values were significantly higher at $12 \mathrm{hpi}$ and significantly lower at $48 \mathrm{hpi}$ in G3 fish, while no significant 
difference was seen between G2 vs. G4 fish. When comparing the percentage of lymphocytes of G3 vs. G4 fish, the values were significantly lower in G4 fish at 0 hpi, but significantly higher at $24 \mathrm{hpi}$, and overall the percentage of lymphocytes seemed very variable between these two groups during the challenge.

The percentage of thrombocytes did not differ in the four groups of fish, except at 12 hpi when the value of G4 fish was significantly higher than that of the other groups.

\subsubsection{Parameters in plasma}

The levels of lysozyme and peroxidase activity and total protein levels in plasma of fish over the duration of the study are shown in Figure 4. High levels of fluctuation could be seen in lysozyme activity and no significant differences at any time-point, except at $3 \mathrm{hpi}$, when the activity in G4 fish was significantly higher than that of G3 fish. For peroxidase activity, significantly higher levels were seen in G3 fish than that of G1 fish at $48 \mathrm{hpi}$, and significantly higher levels were seen in G4 fish compared to G2 fish at 12, 24 and 48 hpi. Between G3 vs. G4 fish, the peroxidase activity was significantly higher in G4 fish at 12 and $24 \mathrm{hpi}$, but significantly lower at $48 \mathrm{hpi}$. The results, therefore, suggested a general increase in the level of plasma peroxidase activity after $S$. agalactiae challenge, both in the Fno-free and Fno-infected fish, and the response in the Fno-infected fish was more than that of the Fno-free fish during 12 and 24 hpi, but had a lower response thereafter.

For plasma protein levels, significantly higher levels of protein were seen in the G3 fish than the G1 fish at 48 hpi, while significantly higher levels were seen in G4 when compared to the G2 fish at 12 hpi. Comparing G3 vs. G4 fish, the protein level in G4 fish was significantly higher than that of G3 fish at $12 \mathrm{hpi}$. The protein level was, therefore, increased by the $S$. agalactiae infection and the response in the Fno-infected fish was earlier (12 hpi) than that of the Fno-free fish (24 hpi). Again, the response of the Fno-infected fish seemed to decline after $24 \mathrm{hpi}$, while that of the Fno-free fish continued to rise at $48 \mathrm{hpi}$. 


\subsubsection{Head-kidney macrophage activities}

Head-kidney macrophage function was also examined in the four groups of fish. For RBA activities and ROS stimulation index without PMA stimulation, these values were significantly higher in G3 fish than those measured in the G1 fish at 12 and 48 hpi, while values in the G4 fish were significantly higher than those of the G2 fish at 12, 24 and 48 hpi (Fig. 5). When values between the G3 vs. G4 fish were compared, the levels produced by macrophages from G4 fish were significantly higher than those from G3 fish at 24 and 48 hpi. The response was similar in the presence of PMA stimulation, except higher values were obtained. The results therefore suggest that the RBA increased in both Fno-free and Fnoinfected fish in response to $S$. agalactiae infection and this response was significantly higher in the Fno-infected fish in 24-48 hpi. The ROS stimulation index was, however, comparable in all groups at all time points, except at $48 \mathrm{~h}$, where the level of G4 fish was significantly lower than those of G2 fish.

Generally, the phagocytic activity of the head-kidney phagocytes was not significantly different in any of the groups of fish, except at 24 hpi when the levels in the G4 fish were significantly higher than that seen in the G3 fish (Fig. 6). The phagocytic index was also similar among all groups at all time-points. When comparing the phagocytic index between G1 and G3 fish, there was a significantly higher activity in the G3 fish than that of G1 fish at 12 hpi. For G2 vs. G4 fish, the latter had a significantly higher index at 24 hpi. Between G1 vs. G2 fish, the phagocytic index of G2 fish was significantly higher than that of G1 fish at 3 hpi, while no significant difference was seen between the G3 vs. G4 fish at any time-point.

Phagocytic capacity, which is the percentage of the fish macrophages that could phagocytise $1(\mathrm{PC} 1)$ or more (PC3, PC5 and $\mathrm{PC} \geq 6$ ) yeast cells, is shown in Figure 7. It can be seenthat most of the macrophages were able to phagocytise 1 yeast cell, i.e., PC1. During 
the challenge, the capacity of phagocytosis of any number of yeast cells between the Fno-free and Fno-infected fish (G3 vs. G4) did not statistically differ, except the PC1 at 3 hpi. At $\mathrm{PC} \geq 6$, the percentage of macrophages from Fno-infected fish was significantly higher than that of Fno-free fish (G1 vs. G2) at 3 hpi, however, by 24 hpi the reverse situation was seen.

Overall, the function of the head-kidney macrophages in their phagocytic abilities (Figs. 6 and 7) in the Fno-free and Fno-infected fish did not seem to be different.

Separate rounds of PCA analysis were run for each time point in this study at $0,3,6$, 12, 24 and 48 hpi (Fig. 8). The component loadings for the 24 parameters [Hct, MCV, RBC count, WBC count, differential WBC count (monocytes, lymphocytes, eosinophils and thrombocytes), RBA (with and without PMA), ROS, lysozyme activity, phagocytic activity, phagocytic index, phagocytic capacity (PC1, PC2, PC3, PC4, PC5, PC6 and PC6+), total protein and plasma peroxidase] were used in each PCA analysis (Supplementary Tables 2 and 3). The component loadings highlight which parameters had a major effect in separating the fish through each principal axis. From this, in general, RBA, RBA with PMA and Hct were the key parameters separating specimens through the first principal component at 12-48 hpi, while total protein, thrombocytes and lymphocytes, among others were the key variables acting through the second principal component. The percentage of the variance explained by the first two factors in each graph are $36.08 \%, 41.79 \%, 35.04 \%$ and $35.82 \%$ respectively (Fig. 8). Significant differences between the groups tested with a one-way ANOVA were seen at $12\left(\mathrm{p}=3.042 \times 10^{-5}\right), 24(\mathrm{p}=0.0004)$ and $48\left(\mathrm{p}=2.40 \times 10^{-6}\right)$ hpi. Bonferroni and Holm post-hoc multiple comparison tests between the pairs of test groups are summarised in Table 2.

\section{Discussion}

Before $S$. agalactiae challenge, the haemato-immunological parameters measured in the Fno-infected red tilapia were comparable to values obtained for the Fno-free fish, except 
for a lower percentage of lymphocytes from the differential white blood cell count. Following S. agalactiae challenge, both the Hct and RBC counts were significantly lower in the Fnoinfected fish, compared to the Fno-free fish. The MCV values of the Fno-free fish, therefore, suggest that the reduced Hct was caused by the reduced number of RBCs, not the size of the RBCs. As the head-kidney is also the site of RBC production [8], it is possible that Fno infection affected erythropoiesis in the Fno-infected fish.

The total WBC and WBC differential counts were similar between the Fno-infected and Fno-free fish before $S$. agalactiae challenge, but were significantly higher in the Fnoinfected fish after the S. agalactiae challenge. This $40-50 \%$ increase of the total WBC count (compared to levels of both Fno-free and Fno-infected only fish) may reflect the "alert" level of the WBC following the natural Fno-outbreak. The percentage of lymphocytes were found to be lower in the Fno-infected fish than in the controls before challenge, but during the challenge, it remained more or less at the same level as that of the Fno-free fish. The finding suggests that Fno infection in the head-kidney of these fish did not impact on the Fno-free myelopoiesis and, probably, lymphopoiesis functions. The presence of basophils has not been reported in the peripheral blood of the hybrid red tilapia [23], but it has been reported that this type of cell is easily overlooked during the microscopic evaluation of cells [24].

Following $S$. agalactiae challenge, plasma lysozyme and peroxidase activities of the Fno-infected fish were significantly higher than those of the Fno-free fish at certain timepoints. These enzymes are both produced by neutrophils and monocytes [24, 25], so the reason that these two enzymes increased following the challenge was possibly due to an increase in the number and function of the WBCs in response to the $S$. agalactiae infection. Peroxidase combines $\mathrm{H}_{2} \mathrm{O}_{2}$ with $\mathrm{Cl}^{-}$to produce hypochlorite, which plays an important role in destroying bacteria [26].

The total plasma protein level in this study was comparable to values described elsewhere [23]. The main protein component in plasma is albumin and immunoglobulin. It is 
not known which component contributed to the increased total plasma protein levels seen in Fno-infected fish, although it is probable that the immunoglobulin, involved in the adaptive immunological response to $S$. agalactiae, might have increased [27].

While it was anticipated that the Fno-infected fish would have a sub-normal RBA, the head-kidney macrophages from the Fno-infected fish was in fact significantly higher than that of the Fno-free fish, with or without PMA stimulation. In addition, the phagocytic activities were found to be comparable to the Fno-free fish. In fish, as in other animals, RBA is a strong defence mechanism against pathogens, which occurs after phagocytosis, and is associated with an intense oxygen consumption by phagocytes, which causes them to produce superoxide and other reactive compounds, such as hydrogen peroxide and hydroxyl radicals; and together with lysozyme and peroxidase, the radicals kill the engulfed pathogens [26]. The results from the plasma enzymes, RBA and phagocytic activities suggest that the Fnoinfected fish did not show any reduced capacity of their innate immune response. In humans, Francisella tularensis infected neutrophils have been shown to have the ability to evade RBA of the infected cells by preventing NADPH oxidase assembly and the bacteria survived well in the infected cells [28]. This mechanism does not seem to operate in the Fno-infected fish in this study as the RBA was increasing despite the Fno-infection.

Principle component analysis (PCA) was additionally used to simultaneously investigate any differences in all the measured parameters, i.e. assessing all 24 variables at each time point. The first PCA analysis showed a high degree of overlap between the four groups of fish at $0 \mathrm{hpi}$, suggesting that the immune parameters did not affect a significant shift in the immune system between the experimental groups. There were, however, significant differences in the immune profiles of fish between G3 and G4 at 12 hpi. At 12, 24 and 48 hpi, significant difference was seen between the non-Streptococcus challenged groups, G1 and G2, and the two Streptococcus-challenged groups, G3 and G4, at 12, 24 and 48 hpi. 
It is interesting to look in more detail as to why the Fno-infected fish had a higher rate of mortality than the normal fish following $S$. agalactiae challenge despite comparable, or even better, innate immune activity.

The first possibility is that the innate immune defence mechanisms of the fish are not adequate to protect the fish from pathogens. It has been shown for Senegalese sole (Solea senegalensis) that increased RBA by their phagocytesis not necessarily protect the fish from Photobacterium damselae infection [29]. In O. niloticus, it has been shown that the correlation between the levels of RBA and protection against a pathogen is not always demonstrated. Two different strains of $O$. niloticus responded differently to Vibrio parahaemolyticus challenge by showing significantly different levels of RBA, but yet had the same mortality rate following challenge with the bacterium [30]. In the current study, however, it was found that normal and Fno-infected fish had different levels of cumulative mortality following infection with $S$. agalactiae, but had similar haematological and innate immune responses. Data from the current study did not show any solid evidence to explain why the Fno-infected fish had higher rates of mortality than the normal fish upon $S$. agalactiae challenge.

The second possibility is that the combination of the Fno and $S$. agalactiae infections resulted in a further decrease in erythropoiesis resulting in acute anemia, with Hct dropping from an average of $26.6 \%$ to $20.6 \%$ within $48 \mathrm{~h}$. The bacteria $S$. agalactiae has been reported to infect several organs of Nile and red tilapia, especially their brain and kidney [14, 31], while in the current study S. agalactiae were cultured from the head-kidney of infected fish.

The third possibility is that Fno-infected fish had a less effective adaptive immune reponse than the normal fish. The production of antibodies is accomplished by lymphocytes, which differentiate into plasma cells after encountering their target antigen. In this study, the percentage of lymphocytes in the Fno-infected fish was not lower than that of the normal fish. The lack of specific antibody production, if any, in this case cannot be due to an 
unavailability of lymphocytes. Interestingly, the head-kidney of many fish, including $O$. niloticus, also contains thyroid hormone-producing cells [32]. As thyroid hormones and the immune system interact and support each other in ways that are still unclear, possible damage to thyroid function by Fno infection in the head-kidney of the fish might be related to the lower resistance against the superimposing $S$. agalactiae infection and this is an interesting point to consider in future research.The impact of other factors imposing additional stress on the host may alter the host's susceptibility to infection can not be ruled out. Reductions in vaccine efficiency have, for example, been seen in fish co-infected with parasites [33, 34].

\section{Conclusions}

Hybrid red tilapia, Oreochromis sp., chronically infected with Fno have normal haematological and innate immune functions and survive well under normal conditions. They, however, have more rapid and higher levels of mortality, compared to the non-infected red tilapia, when experimentally infected with $S$. agalactiae, despite having normal or higher innate immune responses. It is unclear as to why the Fno-infected red tilapia were more susceptible to the $S$. agalactiae infection as their innate immune responses appeared similar to the non Fno-infected tilapia. The Fno-infected fish had an acute reduction in their red blood cell count. Whether the anaemic condition could contribute to the rapid death of the fish requires further study, however, this study showed that the Fno-infected fish had no defect in their innate immunological responses, and thus defective innate immunological function could be ruled out as the possible cause.

\section{Acknowledgements}

This work has been financial supported by The Thailand Research Fund (TRF). The experimental facilities were provided by Veterinary Aquatic Animal Research Health Care Unit, Faculty of Veterianary Science, Mahidol University. The authors would like to thank all 
the staff within the Veterinary Aquatic Animal Research Health Care Unit for their assistance with components of this study.

\section{References}

[1] E. Soto, J. Hawke, D. Fernandez, J. A Morales, Francisella sp., an emerging pathogen of tilapia, Oreochromis niloticus (L.), in Costa Rica, Journal of Fish Diseases 32(8) (2009) $713-722$.

[2] V.V. Nguyen, H.T. Dong, S. Senapin, N. Pirarat, C. Rodkhum, Francisella noatunensis subsp. orientalis, an emerging bacterial pathogen affecting cultured red tilapia (Oreochromis sp.) in Thailand, Aquaculture Research 47(11) (2016) 3697-3702.

[ 3 ] K. Ottem, A. Nylund, E. Karlsbakk, A. Friis-Møller, T. Kamaishi, Elevation of Francisella philomiragia subsp. noatunensis to Francisella noatunensis comb. nov. [syn. Francisella piscicida Ottem et al. (2008) syn. nov.] and characterization of Francisella noatunensis subsp. orientalis subsp. nov., two important fish pathogens, Journal of Applied Microbiology 106(4) (2009) 1231-1243.

[4] K.R. Jeffery, D. Stone, S.W. Feist, D.W. Verner-Jeffreys, An outbreak of disease caused by Francisella sp. in Nile tilapia Oreochromis niloticus at a recirculation fish farm in the UK, Diseases of Aquatic Organisms 91(2) (2010) 161-165.

[5] S. Sridhar, A. Sharma, H. Kongshaug, F. Nilsen, I. Jonassen, Whole genome sequencing of the fish pathogen Francisella noatunensis subsp. orientalis Toba0 4 gives novel insights into Francisella evolution and pathogenecity, BMC Genomics 13(1) (2012) 598.

[6] E. Soto, D. Fernandez, R. Thune, J.P. Hawke, Interaction of Francisella asiatica with tilapia (Oreochromis niloticus) innate immunity, Infection and Immunity 78(5) (2010) 2070-2078.

[7] E. Soto, S. Kidd, S. Mendez, D. Marancik, F. Revan, D. Hiltchie, A. Camus, Francisella noatunensis subsp. orientalis pathogenesis analyzed by experimental immersion 
challenge in Nile tilapia, Oreochromis niloticus (L.), Veterinary Microbiology 164(1-2) (2013) 77-84.

[ 8 ] E.-S.H. Abdel-Aziz, S.B. Abdu, T.E.-S. Ali, H.F. Fouad, Haemopoiesis in the head kidney of tilapia, Oreochromis niloticus (Teleostei: Cichlidae): a morphological (optical and ultrastructural) study, Fish Physiology and Biochemistry 36(3) (2010) 323-336.

[9] E. Soto, K. McGovern-Hopkins, R. Klinger-Bowen, B.K. Fox, J. Brock, N. Antonio, Z. Waal, S. Rushton, A. Mill, C.S. Tamaru, Prevalence of Francisella noatunensis subsp. orientalis in cultured tilapia on the island of Oahu, Hawaii, Journal of Aquatic Animal Health 25(2) (2013) 104-109.

[ 10 ] P.J. Pradeep, R. Suebsing, S. Sirithammajak, J. Kampeera, W. Turner, A. Jeffs, W. Kiatpathomchai, B. Withyachumanarnkul, Vertical transmission and concurrent infection of multiple bacterial pathogens in naturally infected red tilapia (Oreochromis spp.), Aquaculture Research 48(6) (2017) 2706-2717.

[11] M. Amal, M. Zamri-Saad, Streptococcosis in tilapia (Oreochromis niloticus): a review, Pertanika Journal of Tropical Agricultural Science 34(2) (2011) 195-206.

[ 11 2 $]$ ] R. Suebsing, J. Kampeera, B. Tookdee, B. Withyachumnarnkul, W. Turner, W. Kiatpathomchai, Evaluation of colorimetric loop-mediated isothermal amplification assay for visual detection of Streptococcus agalactiae and Streptococcus iniae in tilapia, Letters in Applied Microbiology 57(4) (2013) 317-324.

[13] J. Sun, W. Fang, B. Ke, D. He, Y. Liang, D. Ning, H. Tan, H. Peng, Y. Wang, Y. Ma, Inapparent Streptococcus agalactiae infection in adult/commercial tilapia, Scientific Reports 6 (2016) 26319.

[ 1 l 4 ] G. Assis, G. Tavares, F. Pereira, H. Figueiredo, C. Leal, Natural coinfection by Streptococcus agalactiae and Francisella noatunensis subsp. orientalis in farmed Nile tilapia (Oreochromis niloticus L.), Journal of Fish Diseases 40(1) (2017) 51-63. 
[1 5 ] X. Ye, J. Li, M. Lu, G. Deng, X. Jiang, Y. Tian, Y. Quan, Q. Jian, Identification and molecular typing of Streptococcus agalactiae isolated from pond-cultured tilapia in China, Fisheries Science 77(4) (2011) 623-632.

[16] W. Sirimanapong, K.D. Thompson, K. Kledmanee, P. Thaijongrak, B. Collet, E.L. Ooi, A. Adams, Optimisation and standardisation of functional immune assays for striped catfish (Pangasianodon hypophthalmus) to compare their immune response to live and heat killed Aeromonas hydrophila as models of infection and vaccination, Fish \& Shellfish Immunology 40(2) (2014) 374-383.

[17] A.L. Morgan, K. Thompson, N. Auchinachie, H. Migaud, The effect of seasonality on normal haematological and innate immune parameters of rainbow trout Oncorhynchus mykiss L, Fish \& Shellfish Immunology 25(6) (2008) 791-799.

[ 1 r 8 ] M.J. Quade, J.A. Roth, A rapid, direct assay to measure degranulation of bovine neutrophil primary granules, Veterinary Immunology and Immunopathology 58 ( 3 -4 ) (1997) 239-248.

[19] A. Sitjà-Bobadilla, S. Peña-Llopis, P. Gómez-Requeni, F. Médale, S. Kaushik, J. PérezSánchez, Effect of fish meal replacement by plant protein sources on non-specific defence mechanisms and oxidative stress in gilthead sea bream (Sparus aurata), Aquaculture 249(1-4) (2005) 387-400.

[20] W. Sirimanapong, K.D. Thompson, E.L. Ooi, M. Bekaert, B. Collet, J.B. Taggart, J.E. Bron, D.M. Green, A.P. Shinn, A. Adams, The effects of feeding $\beta$-glucan to Pangasianodon hypophthalmus on immune gene expression and resistance to Edwardsiella ictaluri, Fish \& Shellfish Immunology 47(1) (2015) 595-605.

[21] V. Findlay, B. Munday, The immunomodulatory effects of levamisole on the nonspecific immune system of Atlantic salmon, Salmo salar L, Journal of Fish Diseases 23(6) (2000) 369-378. 
[ 2 2 2 ] N N.B. Buller, Bacteria and fungi from fish and other aquatic animals: a practical identification manual, Cabi 2014.

[23 ] T.C. Hrubec, J.L. Cardinale, S.A. Smith, Hematology and plasma chemistry reference intervals for cultured tilapia (Oreochromis hybrid), Veterinary Clinical Pathology 29(1) (2000) 7-12.

[2 4 ] I.K. Ueda, M.I. Egami, W.d.S. Sasso, E.R. Matushima, Cytochemical aspects of the peripheral blood cells of Oreochromis (Tilapia) niloticus (Linnaeus, 1758 ) (Cichlidae, Teleostei): part II, Brazilian Journal of Veterinary Research and Animal Science 38 (6) (2001) 273-277.

[25] G. Scapigliati, N. Romano, F. Buonocore, S. Picchietti, M. Baldassini, D. Prugnoli, A. Galice, S. Meloni, C.J. Secombes, M. Mazzini, The immune system of sea bass, Dicentrarchus labrax, reared in aquaculture, Developmental \& Comparative Immunology 26(2) (2002) 151-160.

[26] C.J. Secombes, T. Fletcher, The role of phagocytes in the protective mechanisms of fish, Annual Review of Fish Diseases 2 (1992) 53-71.

[27] R. Harikrishnan, C. Balasundaram, M.-S. Heo, Lactobacillus sakei BK19 enriched diet enhances the immunity status and disease resistance to streptococcosis infection in kelp grouper, Epinephelus bruneus, Fish \& Shellfish Immunology 29(6) (2010) 1037-1043.

[2 8 ] R.L. McCaffrey, L.A.H. Allen, Francisella tularensis LVS evades killing by human neutrophils via inhibition of the respiratory burst and phagosome escape, Journal of Leukocyte Biology 80(6) (2006) 1224-1230.

[29] P. Díaz-Rosales, S. Arijo, M. Chabrillón, F. Alarcón, S. Tapia-Paniagua, E. MartínezManzanares, M. Balebona, M. Moriñigo, Effects of two closely related probiotics on respiratory burst activity of Senegalese sole (Solea senegalensis, Kaup) phagocytes, and protection against Photobacterium damselae subsp. piscicida, Aquaculture 293(1-2) (2009) 16-21. 
[30] S. Balfry, M. Shariff, G.K. Iwama, Strain differences in non-specific immunity of tilapia Oreochromis niloticus following challenge with Vibrio parahaemolyticus, Diseases of Aquatic Organisms 30(1) (1997) 77-80.

[3 1] E. Hernández, J. Figueroa, C. Iregui, Streptococcosis on a red tilapia, Oreochromis sp., farm: a case study, Journal of Fish Diseases 32(3) (2009) 247-252.

[ 3 2 2 ] E.J. Geven, P.H. Klaren, The teleost head kidney: Integrating thyroid and immune signalling, Developmental \& Comparative Immunology 66 (2017) 73-83.

[3 3 ] M.L. Martins, C.A. Shoemaker, D. Xu, P.H. Klesius, Effect of parasitism on vaccine efficacy against Streptococcus iniae in Nile tilapia, Aquaculture 314(1-4) (2011) 18-23.

[3 4 ] J.F. Urban Jr, N.R. Steenhard, G.I. Solano-Aguilar, H.D. Dawson, O.I. Iweala, C.R. Nagler, G.S. Noland, N. Kumar, R.M. Anthony, T. Shea-Donohue, Infection with parasitic nematodes confounds vaccination efficacy, Veterinary parasitology 148 (1) (2007) 14-20. 
Table 1. Haemato-immunological parameters of hybrid red tilapia compared between Fnofree and Fno-infected fish.

\begin{tabular}{|c|c|c|}
\hline & Fno-free & Fno-infected \\
\hline \multicolumn{3}{|l|}{ Haematology } \\
\hline Haematocrit (\%) & $29.0 \pm 3.1$ & $27.2 \pm 3.6$ \\
\hline Total $\mathrm{RBC}$ count $\left(\times 10^{6}\right.$ cells $\left./ \mu \mathrm{L}\right)$ & $1.68 \pm 0.48$ & $1.58 \pm 0.31$ \\
\hline Mean corpuscular volume (fL) & $189.8 \pm 71.0$ & $178.3 \pm 41.2$ \\
\hline Total WBC count $\left(\times 10^{4}\right.$ cells $\left./ \mu \mathrm{L}\right)$ & $1.41 \pm 0.40$ & $1.43 \pm 0.19$ \\
\hline \multicolumn{3}{|l|}{ WBC differential count (\%) } \\
\hline Neutrophils & $21.5 \pm 4.5$ & $24.3 \pm 6.0$ \\
\hline Eosinophils & $3.1 \pm 1.9$ & $3.5 \pm 2.8$ \\
\hline Monocytes & $2.3 \pm 0.8$ & $3.0 \pm 1.9$ \\
\hline Lymphocytes & $42.7 \pm 3.3$ & $36.4 \pm 7.5^{*}$ \\
\hline Thrombocytes & $30.4 \pm 6.9$ & $32.8 \pm 7.1$ \\
\hline \multicolumn{3}{|l|}{ Innate immune response } \\
\hline Lysozyme activity (unit/mL) & $257.9 \pm 110.8$ & $266.2 \pm 165.3$ \\
\hline Peroxidase activity (450-nm absorbance) & $3.7 \pm 0.9$ & $3.6 \pm 0.7$ \\
\hline Total protein $(\mathrm{mg} / \mathrm{mL})$ & $322 \pm 88$ & $320 \pm 30$ \\
\hline \multicolumn{3}{|l|}{ Respiratory burst activity } \\
\hline Reactive oxygen species index & $1.88 \pm 0.35$ & $1.97 \pm 0.39$ \\
\hline \multicolumn{3}{|l|}{ Phagocytic activity } \\
\hline Phagocytic activity (\%) & $90.3 \pm 1.7$ & $89.8 \pm 4.1$ \\
\hline Phagocytic index (yeast cells/phagocyte) & $3.5 \pm 0.2$ & $3.5 \pm 0.3$ \\
\hline \multicolumn{3}{|l|}{ Phagocytic capacity (\%) } \\
\hline 1-yeast cell/phagocyte & $14.4 \pm 2.9$ & $16.2 \pm 4.0$ \\
\hline 2-yeast cell/phagocyte & $20.3 \pm 5.3$ & $15.9 \pm 3.7$ \\
\hline 3 -yeast cell/phagocyte & $13.8 \pm 4.1$ & $12.6 \pm 2.7$ \\
\hline 4-yeast cell/phagocyte & $12.1 \pm 4.2$ & $13.5 \pm 2.7$ \\
\hline 5-yeast cell/phagocyte & $6.0 \pm 1.6$ & $6.2 \pm 0.8$ \\
\hline 6-yeast cell/phagocyte & $4.5 \pm 1.2$ & $5.1 \pm 0.9$ \\
\hline$>6$-yeast cell/phagocyte & $5.5 \pm 1.6$ & $5.5 \pm 1.4$ \\
\hline
\end{tabular}

Fno, Francisella noatunensis; RBC, red blood cell; WBC, white blood cell. * $p<0.05$; ** (610-nm absorbance for $10^{6}$ cells); Values represent the mean value of 10 fish \pm SD 
Table 2. Bonferroni and Holm post-hoc tests ran on the factor scores for the first principal component derived from each analysis testing for statistical significance $(p<0.05)$. Where G1 $=$ Fno-free fish that were not challenged with S. agalactiae; G2 =Fno-infected fish that were not challenged with $S$. agalactiae; G3 = Fno-free fish that were challenged with $S$. agalactiae; and, G4 = Fno-infected fish that were challenged with S. agalactiae. Fno, Francisella noatunensis; NS, not significant.

\begin{tabular}{ccccccc}
\hline & \multicolumn{2}{c}{ 12 hpi } & \multicolumn{2}{c}{ 24 hpi } & \multicolumn{2}{c}{ 48 hpi } \\
\hline $\begin{array}{c}\text { Treatment } \\
\text { pair }\end{array}$ & Bonferroni & Holm & Bonferroni & Holm & Bonferroni & Holm \\
\hline G1 vs G2 & NS & NS & NS & NS & NS & NS \\
G1 vs G4 & $p<0.01$ & $p<0.01$ & $p<0.01$ & $p<0.01$ & $p<0.01$ & $p<0.01$ \\
G1 vs G3 & $p<0.01$ & $p<0.01$ & NS & NS & $p<0.01$ & $p<0.01$ \\
G2 vs G4 & $p<0.01$ & $p<0.01$ & $p<0.01$ & $p<0.01$ & $p<0.01$ & $p<0.01$ \\
G2 vs G3 & $p<0.01$ & $p<0.01$ & NS & $p<0.05$ & $p<0.01$ & $p<0.01$ \\
G4 vs G3 & NS & $p<0.05$ & NS & NS & NS & NS \\
\hline
\end{tabular}


Supplementary Table 1. Differential white blood cell counts of Fno (Francisella noatunensis) -free and Fno-infected red tilapia

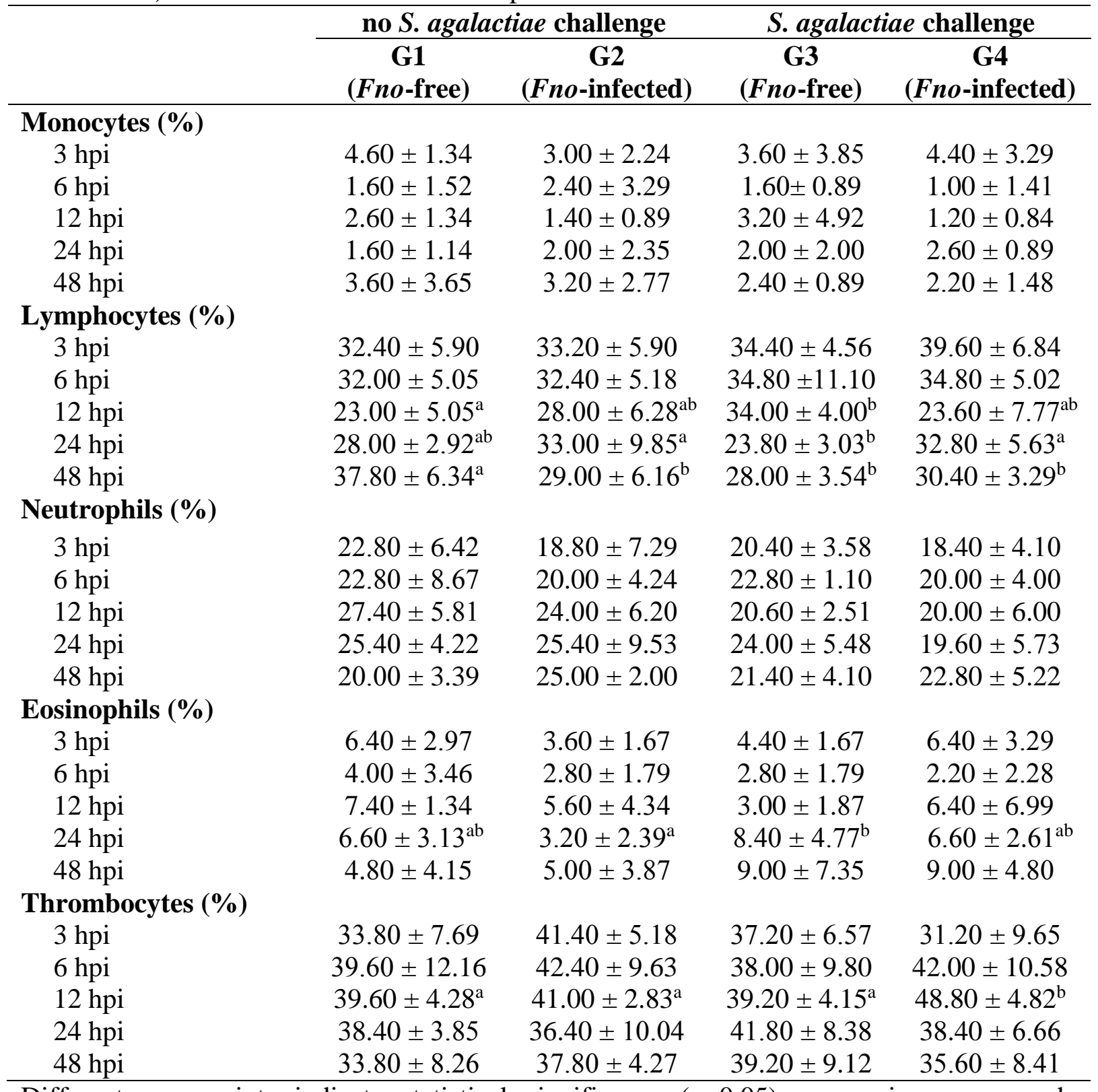

Different superscripts indicate statistical significance $(p<0.05)$; comparison was made between the values of the same row. Values represent the mean value of 5 fish \pm SD. 
Supplementary Table 2. The component loadings and the percentage of the variance explained by each variable $(\mathrm{n}=24)$ for each principal components analysis (PCA). PCA 1 ( $t=0$ hour post-infection (p.i.); 4 groups; $\mathrm{n}=5$ replicates per group); PCA 2 ( $t=3$ hpi.); PCA 3 ( $t=6$ hpi.). Each analysis considers four groups of samples: G1 - Fno-free fish not subjected to a Streptococcus agalactiae challenge; G2 - Fno-infected fish not subjected to a $S$. agalactiae challenge; G3 Fno-free fish subjected to an S. agalactiae challenge and G4 - Fno-infected fish subjected to a $S$. agalactiae challenge. Values above \pm 0.70 are shown in a bold font. Fno, Francisella noatunensis

\begin{tabular}{|c|c|c|c|c|c|c|c|c|c|}
\hline \multirow[t]{2}{*}{ Variable } & \multicolumn{3}{|c|}{ PCA 1 ( $t=0$ hpi.) } & \multicolumn{3}{|c|}{ PCA 2 ( $t=3$ hpi.) } & \multicolumn{3}{|c|}{ PCA 3 ( $t=6$ hpi.) } \\
\hline & $\begin{array}{c}\text { Factor } \\
1 \\
\end{array}$ & $\begin{array}{c}\text { Factor } \\
\mathbf{2} \\
\end{array}$ & $\begin{array}{c}\text { Factor } \\
\mathbf{3} \\
\end{array}$ & $\begin{array}{c}\text { Factor } \\
1 \\
\end{array}$ & $\begin{array}{c}\text { Factor } \\
\mathbf{2} \\
\end{array}$ & $\begin{array}{c}\text { Factor } \\
3 \\
\end{array}$ & $\begin{array}{c}\text { Factor } \\
1 \\
\end{array}$ & $\begin{array}{c}\text { Factor } \\
\mathbf{2} \\
\end{array}$ & $\begin{array}{c}\text { Factor } \\
\mathbf{3}\end{array}$ \\
\hline HCT & 0.484 & -0.323 & 0.520 & 0.345 & 0.004 & 0.480 & -0.017 & 0.488 & -0.175 \\
\hline MCV & 0.793 & -0.299 & -0.253 & 0.109 & -0.766 & 0.342 & -0.442 & 0.447 & 0.593 \\
\hline $\mathrm{RBC} \times 10^{5} \mathrm{cell} / \mathrm{ml}$ & -0.708 & 0.108 & 0.333 & 0.022 & 0.783 & 0.012 & 0.449 & -0.169 & -0.752 \\
\hline WBC $\times 10^{4}$ cell $/ \mathrm{ml}$ & -0.452 & -0.281 & 0.411 & 0.090 & 0.157 & -0.392 & 0.232 & -0.393 & -0.308 \\
\hline Respiratory burst activity (RBA) & -0.646 & -0.325 & 0.483 & -0.039 & -0.630 & -0.581 & -0.454 & 0.418 & 0.024 \\
\hline Respiratory burst activity (RBA with PMA) & -0.554 & -0.349 & 0.378 & 0.154 & -0.784 & -0.383 & -0.303 & 0.369 & -0.345 \\
\hline Reactive oxygen species (ROS) & 0.157 & -0.087 & -0.188 & 0.763 & -0.176 & 0.259 & 0.344 & -0.234 & -0.663 \\
\hline Lysozyme activity & -0.233 & 0.351 & -0.341 & -0.233 & -0.563 & 0.158 & -0.141 & -0.733 & -0.032 \\
\hline Total protein & -0.591 & -0.216 & -0.354 & -0.377 & 0.346 & 0.262 & 0.313 & 0.100 & 0.290 \\
\hline Plasma peroxidase & 0.176 & -0.477 & 0.522 & -0.664 & -0.172 & -0.151 & -0.278 & -0.635 & -0.254 \\
\hline Monocytes & -0.193 & 0.512 & 0.225 & -0.051 & 0.376 & -0.160 & 0.201 & -0.506 & 0.408 \\
\hline Lymphocytes & 0.017 & -0.297 & 0.403 & -0.187 & -0.155 & -0.553 & -0.641 & -0.515 & 0.133 \\
\hline Neutrophils & -0.777 & 0.163 & -0.072 & -0.316 & 0.139 & 0.050 & -0.272 & -0.244 & 0.215 \\
\hline Eosinophils & -0.222 & 0.239 & -0.297 & -0.036 & 0.678 & -0.342 & -0.020 & -0.233 & 0.060 \\
\hline Thrombocytes & 0.700 & -0.039 & -0.271 & 0.385 & -0.335 & 0.544 & 0.528 & 0.614 & -0.288 \\
\hline Phagocytic activity (PA) & -0.175 & 0.715 & 0.316 & -0.682 & 0.131 & 0.305 & 0.495 & -0.096 & -0.153 \\
\hline Phagocytic index (PI) & 0.066 & 0.889 & 0.264 & -0.939 & -0.050 & 0.192 & 0.896 & 0.025 & 0.234 \\
\hline Phagocytic capacity 1 (PC1) & -0.518 & -0.330 & -0.318 & 0.744 & 0.092 & -0.571 & -0.668 & -0.288 & -0.407 \\
\hline Phagocytic capacity 2 (PC2) & -0.034 & -0.485 & -0.478 & 0.688 & 0.214 & 0.306 & -0.507 & 0.516 & -0.090 \\
\hline Phagocytic capacity 3 (PC3) & 0.352 & 0.031 & 0.665 & 0.177 & 0.366 & 0.267 & -0.420 & 0.023 & -0.563 \\
\hline Phagocytic capacity 4 (PC4) & 0.518 & 0.095 & 0.480 & -0.161 & -0.157 & 0.367 & -0.037 & -0.175 & 0.665 \\
\hline Phagocytic capacity 5 (PC5) & -0.319 & 0.168 & 0.214 & -0.159 & -0.551 & -0.045 & 0.483 & -0.400 & 0.022 \\
\hline Phagocytic capacity 6 (PC6) & -0.595 & 0.052 & -0.207 & -0.339 & -0.089 & 0.623 & 0.501 & -0.572 & 0.273 \\
\hline Phagocytic capacity 6+ (PC6+) & 0.309 & 0.663 & -0.115 & -0.800 & 0.034 & -0.260 & 0.631 & 0.629 & 0.064 \\
\hline$\%$ Total variation & 0.216 & 0.145 & 0.133 & 0.203 & 0.168 & 0.130 & 0.195 & 0.176 & 0.132 \\
\hline Cumulative percentage & 21.59 & 36.08 & 49.38 & 20.34 & 37.16 & 50.2 & 19.49 & 37.09 & 50.24 \\
\hline
\end{tabular}


Supplementary Table 3. The component loadings and the percentage of the variance explained by each variable $(\mathrm{n}=24)$ for each principal components analysis (PCA). PCA $4(t=12$ hpi.); PCA $5(t=24$ hpi.) and PCA $6(t=48$ hpi.). Each analysis considers four groups of samples: G1 Fno-free fish not subjected to a Streptococcus agalactiae challenge; G2 - Fno-infected fish not subjected to a S. agalactiae challenge; G3 - Fno-free fish subjected to an S. agalactiae challenge and G4 - Fno-infected fish subjected to a $S$. agalactiae challenge. Values above \pm 0.70 are shown in a bold font. Fno, Francisella noatunensis

\begin{tabular}{|c|c|c|c|c|c|c|c|c|c|}
\hline \multirow[t]{2}{*}{ Variable } & \multicolumn{3}{|c|}{ PCA 4 ( $t=12$ hpi.) } & \multicolumn{3}{|c|}{ PCA 5 ( $t=24$ hpi.) } & \multicolumn{3}{|c|}{ PCA 6 ( $t=48$ hpi.) } \\
\hline & $\begin{array}{c}\text { Factor } \\
1\end{array}$ & $\begin{array}{c}\text { Factor } \\
2\end{array}$ & $\begin{array}{c}\text { Factor } \\
3\end{array}$ & $\begin{array}{c}\text { Factor } \\
1\end{array}$ & $\begin{array}{c}\text { Factor } \\
2\end{array}$ & $\begin{array}{c}\text { Factor } \\
3\end{array}$ & $\begin{array}{c}\text { Factor } \\
1\end{array}$ & $\begin{array}{c}\text { Factor } \\
2\end{array}$ & $\begin{array}{c}\text { Factor } \\
3\end{array}$ \\
\hline HCT & -0.819 & 0.296 & -0.147 & -0.341 & 0.373 & 0.422 & 0.768 & -0.249 & 0.073 \\
\hline MCV & -0.288 & -0.441 & 0.006 & -0.513 & -0.475 & -0.236 & -0.073 & -0.173 & 0.546 \\
\hline $\mathrm{RBC} \times 10^{5} \mathrm{cell} / \mathrm{ml}$ & -0.367 & 0.563 & -0.104 & 0.204 & 0.627 & 0.406 & 0.471 & 0.082 & -0.427 \\
\hline WBC $\times 10^{4}$ cell $/ \mathrm{ml}$ & 0.614 & 0.555 & 0.053 & 0.585 & -0.286 & 0.391 & -0.607 & 0.014 & 0.178 \\
\hline Respiratory burst activity (RBA) & 0.785 & -0.193 & -0.431 & 0.860 & -0.109 & -0.269 & -0.745 & 0.080 & 0.203 \\
\hline Respiratory burst activity (RBA with PMA) & 0.778 & -0.381 & -0.323 & 0.871 & -0.243 & -0.253 & -0.778 & 0.003 & 0.216 \\
\hline Reactive oxygen species (ROS) & -0.168 & -0.388 & 0.364 & -0.198 & -0.485 & -0.163 & 0.708 & 0.060 & 0.184 \\
\hline Lysozyme activity & 0.086 & 0.079 & -0.240 & -0.430 & 0.026 & 0.115 & 0.571 & -0.028 & 0.171 \\
\hline Total protein & 0.614 & -0.597 & -0.032 & 0.745 & -0.095 & -0.275 & -0.606 & -0.076 & 0.135 \\
\hline Plasma peroxidase & 0.812 & -0.437 & -0.236 & 0.458 & -0.226 & -0.037 & -0.676 & 0.069 & 0.299 \\
\hline Monocytes & -0.280 & -0.031 & -0.735 & 0.544 & -0.290 & 0.259 & 0.332 & 0.729 & -0.094 \\
\hline Lymphocytes & 0.025 & 0.687 & -0.350 & -0.295 & -0.557 & -0.371 & 0.489 & -0.153 & 0.330 \\
\hline Neutrophils & -0.444 & -0.070 & 0.596 & -0.481 & 0.607 & -0.414 & -0.010 & 0.568 & -0.324 \\
\hline Eosinophils & 0.026 & -0.294 & -0.421 & 0.444 & 0.571 & -0.142 & -0.295 & 0.592 & 0.181 \\
\hline Thrombocytes & 0.547 & -0.571 & 0.493 & 0.364 & -0.244 & 0.733 & -0.291 & -0.828 & -0.194 \\
\hline Phagocytic activity (PA) & 0.509 & 0.418 & 0.134 & 0.456 & 0.321 & -0.130 & 0.309 & -0.164 & 0.416 \\
\hline Phagocytic index (PI) & 0.715 & 0.517 & 0.262 & 0.370 & 0.287 & -0.145 & 0.229 & -0.484 & 0.680 \\
\hline Phagocytic capacity 1 (PC1) & -0.671 & -0.263 & -0.251 & -0.015 & 0.274 & 0.461 & -0.267 & 0.430 & -0.288 \\
\hline Phagocytic capacity 2 (PC2) & -0.238 & -0.346 & 0.242 & 0.364 & -0.259 & -0.435 & -0.198 & 0.177 & -0.379 \\
\hline Phagocytic capacity 3 (PC3) & -0.174 & -0.377 & 0.108 & -0.332 & -0.572 & 0.246 & 0.445 & 0.400 & 0.429 \\
\hline Phagocytic capacity 4 (PC4) & -0.511 & -0.162 & -0.357 & -0.174 & 0.331 & -0.759 & -0.066 & -0.389 & -0.290 \\
\hline Phagocytic capacity 5 (PC5) & 0.482 & 0.161 & -0.431 & 0.352 & -0.056 & -0.067 & 0.361 & -0.259 & -0.486 \\
\hline Phagocytic capacity 6 (PC6) & 0.393 & 0.220 & -0.325 & -0.220 & -0.306 & 0.224 & -0.216 & -0.705 & -0.159 \\
\hline Phagocytic capacity $6+($ PC6+) & 0.461 & 0.408 & 0.670 & 0.288 & 0.357 & 0.174 & 0.030 & 0.237 & 0.893 \\
\hline \% Total variation & 0.263 & 0.155 & 0.129 & 0.211 & 0.139 & 0.121 & 0.215 & 0.143 & 0.136 \\
\hline Cumulative percentage & 26.26 & 41.79 & 54.7 & 21.11 & 35.04 & 47.14 & 21.49 & 35.82 & 49.4 \\
\hline
\end{tabular}




\section{Figure legends}

Figure 1. Survival rate of Fno-free and Fno-infected red tilapia following S. agalactiae challenge, compared to that of the non-challenge fish. Values are means \pm standard deviation with $n=24$ fish per tank in five replicate tanks per group. Fno,

Francisella noatunensis.

Figure 2. Haematocrit (2A), total red blood cell count (2B) and mean corpuscular volume (2C) of normal and Fno-infected red tilapia, at time 3, 6, 12, 24 and 48 hours following S. agalactiae challenge. Values are means \pm standard deviation with $n$ $=5$ per group. Statistical comparison was made among the values of the same time-point; different superscripts indicate statistical significance $(p<0.05)$. Fno, Francisella noatunensis.

Figure 3. Total white blood cell count (3A) and percentage of lymphocytes (3B) and thrombocytes (3C) of Fno-free and Fno-infected red tilapia, at time 3, 6, 12, 24 and 48 hours following S. agalactiae challenge. Values are means \pm standard deviation with $n=5$ per group. Statistical comparison was made among the values of the same time-point; different superscripts indicate statistical significance $(p<0.05)$. Fno, Francisella noatunensis.

Figure 4. Activities of plasma lysozyme (4A) and peroxidase (4B) and level of plasma protein (4C) of Fno-free and Fno-infected red tilapia, at time 3, 6, 12, 24 and 48 hours following S. agalactiae challenge. Values are means \pm standard deviation with $n=5$ per group. Statistical comparison was made among the values of the same time-point; different superscripts indicate statistical significance $(p<0.05)$. Fno, Francisella noatunensis.

Figure 5. Respiratory burst activities (RBA), with (5A) and without (5B) phorbol myristate acetate (PMA) stimulation and reactive oxygen species (ROS) stimulation index (5C) of Fno-free and Fno-infected red tilapia, at time 3, 6, 12, 24 and 48 hours 
following S. agalactiae challenge. Values are means \pm standard deviation with $n$ $=5$ per group. Statistical comparison was made among the values of the same time-point; different superscripts indicate statistical significance $(p<0.05)$. Fno, Francisella noatunensis.

Figure 6. Phagocytic activity (6A) and phagocytic index (6B) of Fno-free and Fno-infected red tilapia, at time 3, 6, 12, 24 and 48 hours following $S$. agalactiae challenge. Values are means \pm standard deviation with $n=5$ per group. Statistical comparison was made among the values of the same time-point; different superscripts indicate statistical significance $(p<0.05)$. Fno, Francisella noatunensis.

Figure 7. Phagocytic capacity of Fno-free and Fno-infected red tilapia, at time 3, 6, 12, 24 and 48 hours following S. agalactiae challenge. Values are means \pm standard deviation with $n=5$ per group. Statistical comparison was made among the values of the same time-point; different superscripts indicate statistical significance ( $p<0.05)$. PC1 (7A), PC3 (7B), PC5 (7C) and PC $\geq 6$ (7D) signify the percentage of phogocytes containing 1 yeast cell or more. Fno, Francisella noatunensis.

Figure 8. Principal components analysis of the 24 hemato-immunological parameters measured from each group of fish $(\mathrm{n}=5)$ at $0,12,24$ and 48 hours post-infection. For each PCA plot, the first two principal components (Factor 1 vs Factor 2) accounting for the most variation between the specimens are shown. The variables making a major contribution to the separation of the specimens through each Factor and the direction in which they act are marked. Fno, Francisella noatunensis. 
Figure 1

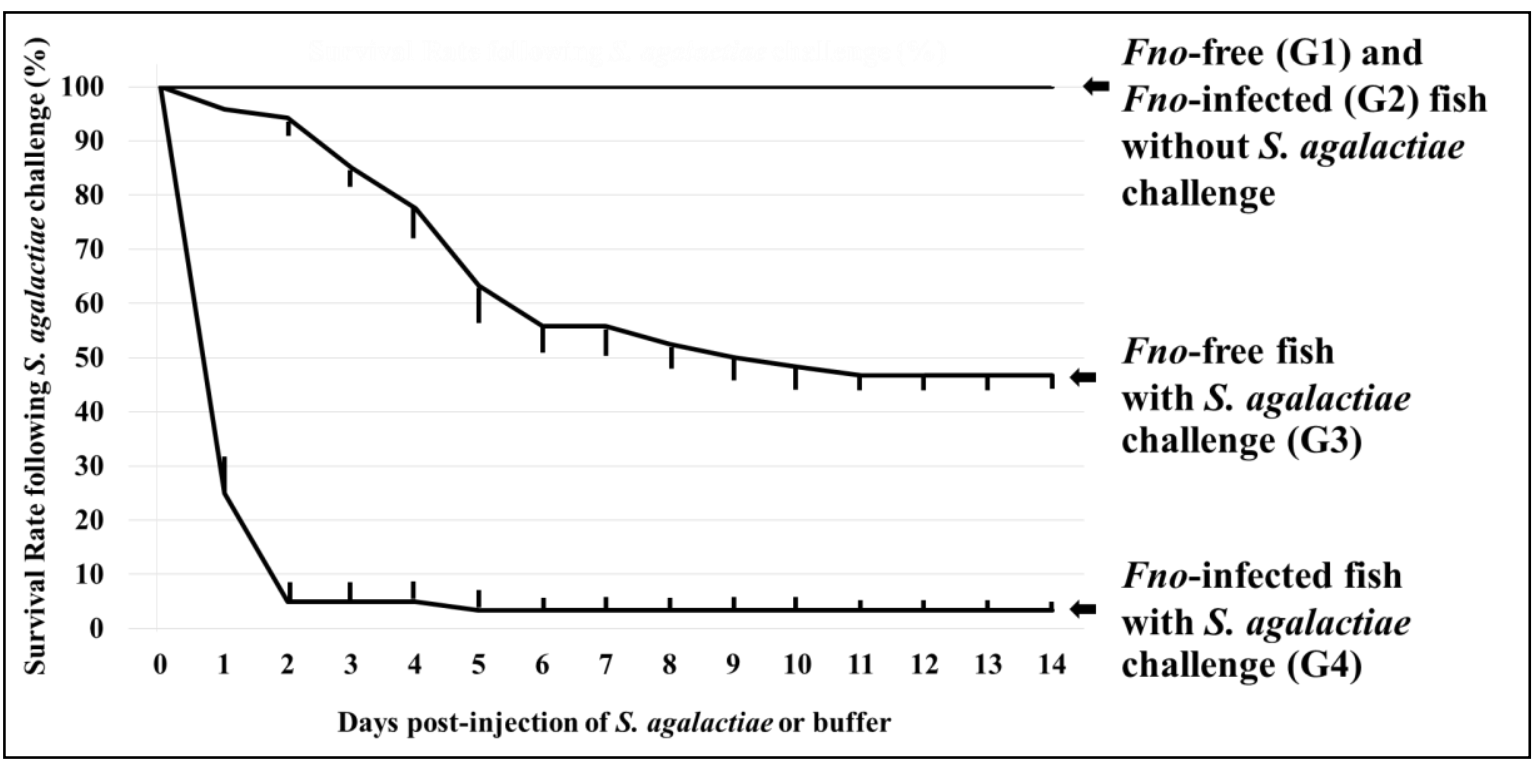


Figure 2
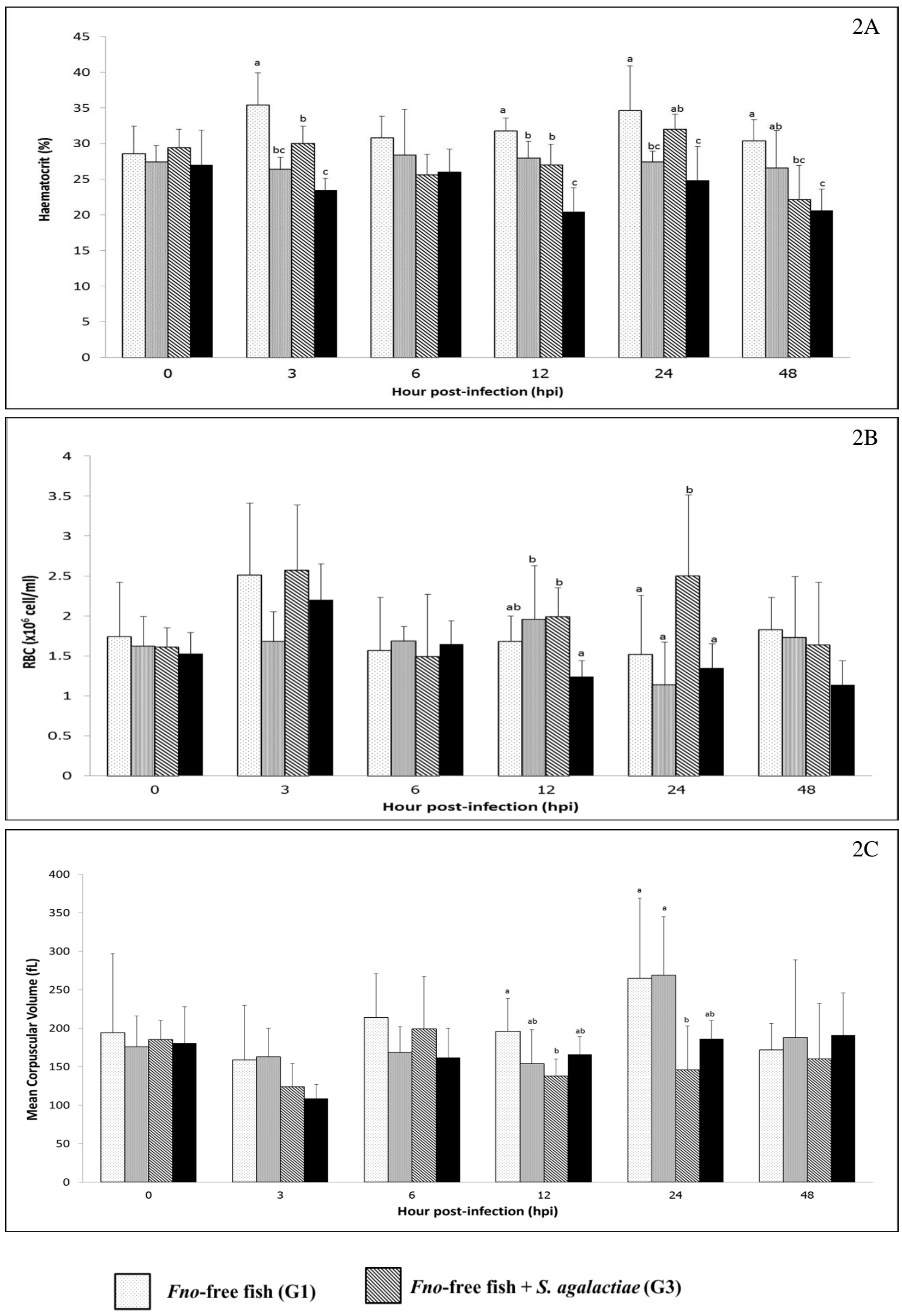

Fno-free fish (G1)

Fno-free fish + S. agalactiae (G3)

Fno-infected fish (G2)

Fno-infected fish + S. agalactiae (G4) 
Figure 3
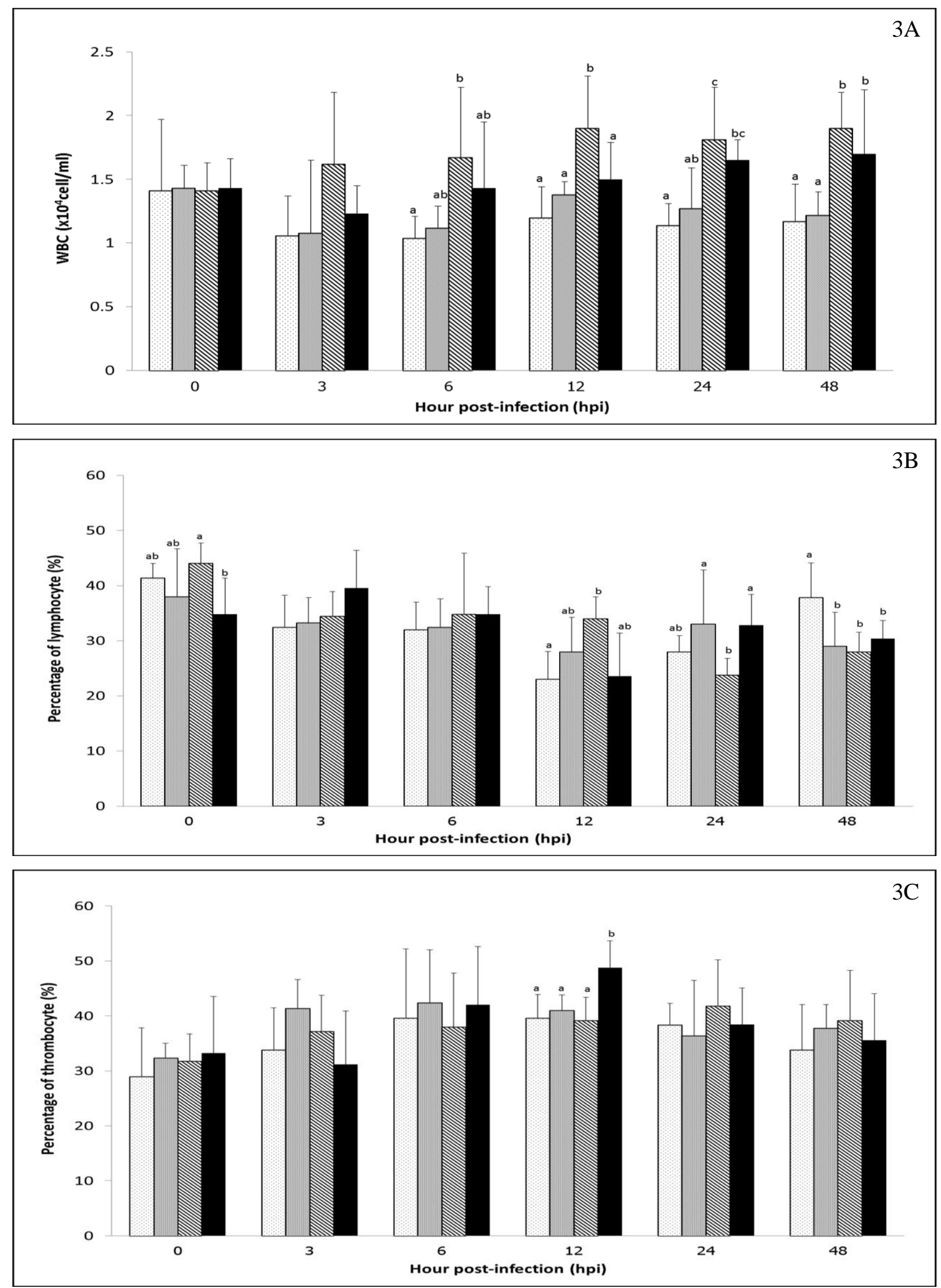

Fno-free fish (G1)

Fno-free fish + S. agalactiae (G3)

Fno-infected fish (G2)

Fno-infected fish + S. agalactiae (G4) 
Figure 4
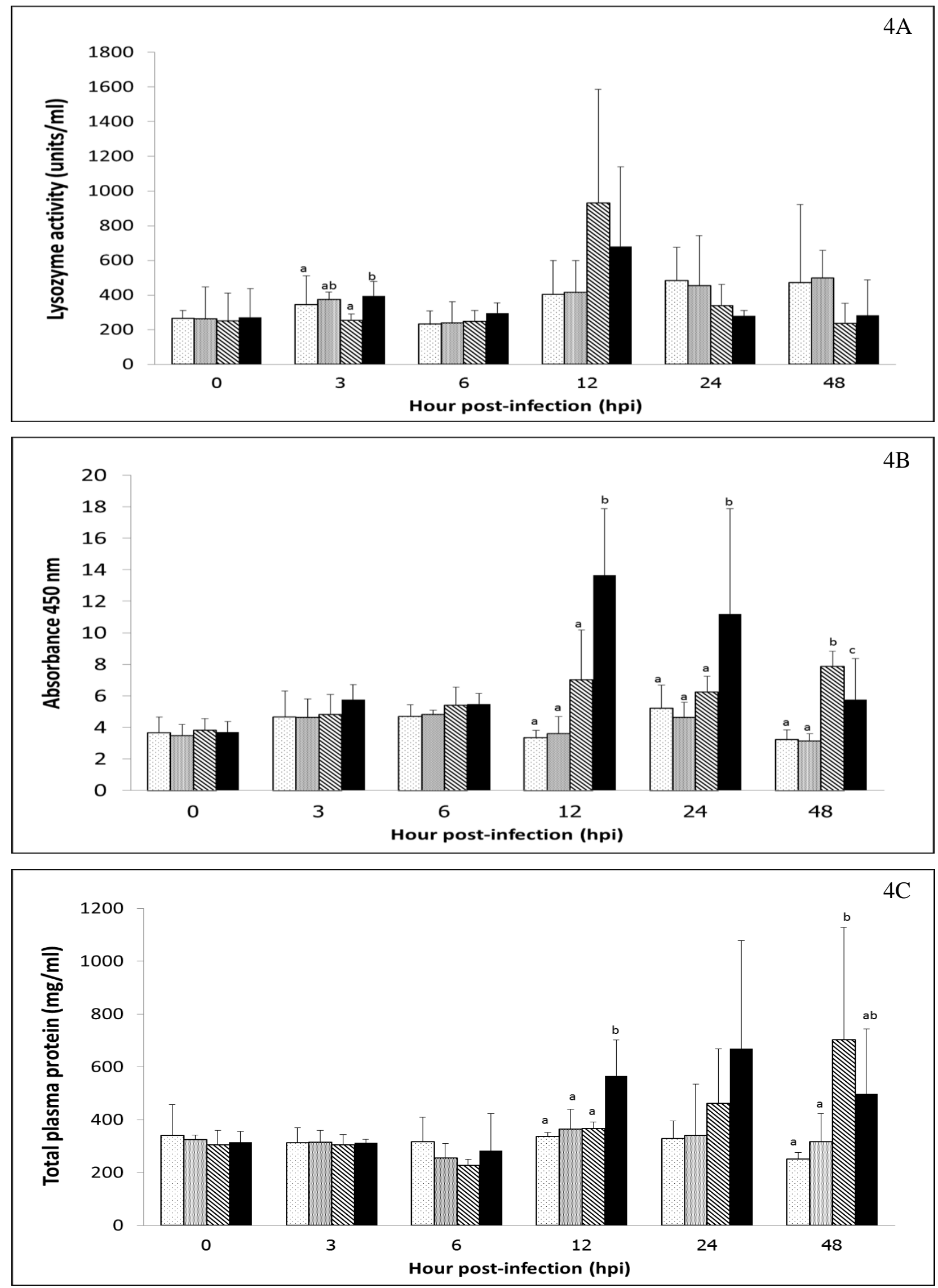

Fno-free fish (G1)

Fno-free fish + S. agalactiae $(\mathrm{G} 3)$

Fno-infected fish (G2)

Fno-infected fish $+S$. agalactiae $(\mathrm{G} 4)$ 
Figure 5
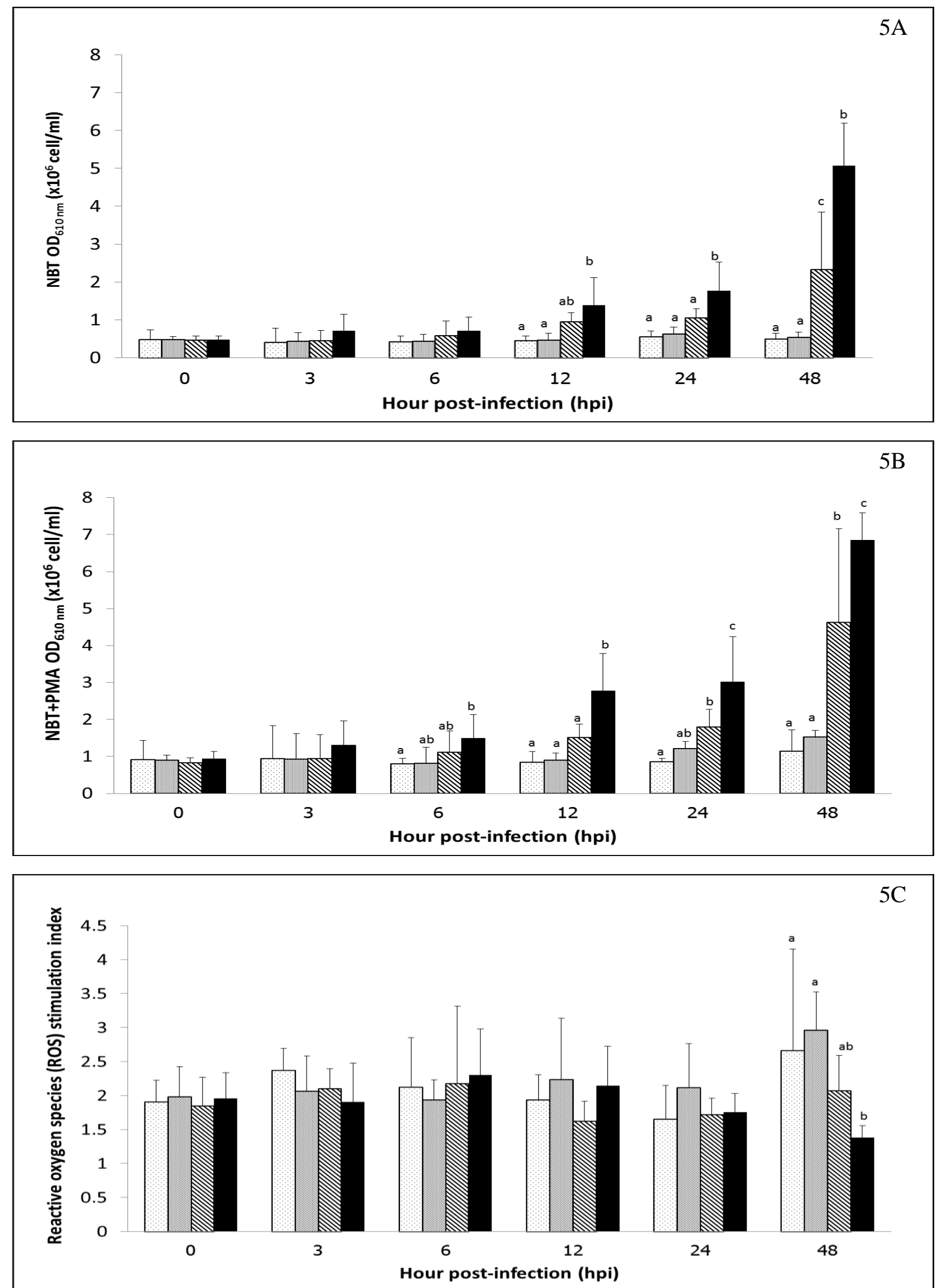

Fno-free fish (G1)

Fno-free fish + S. agalactiae (G3)

Fno-infected fish (G2)

Fno-infected fish + S. agalactiae (G4) 
Figure 6

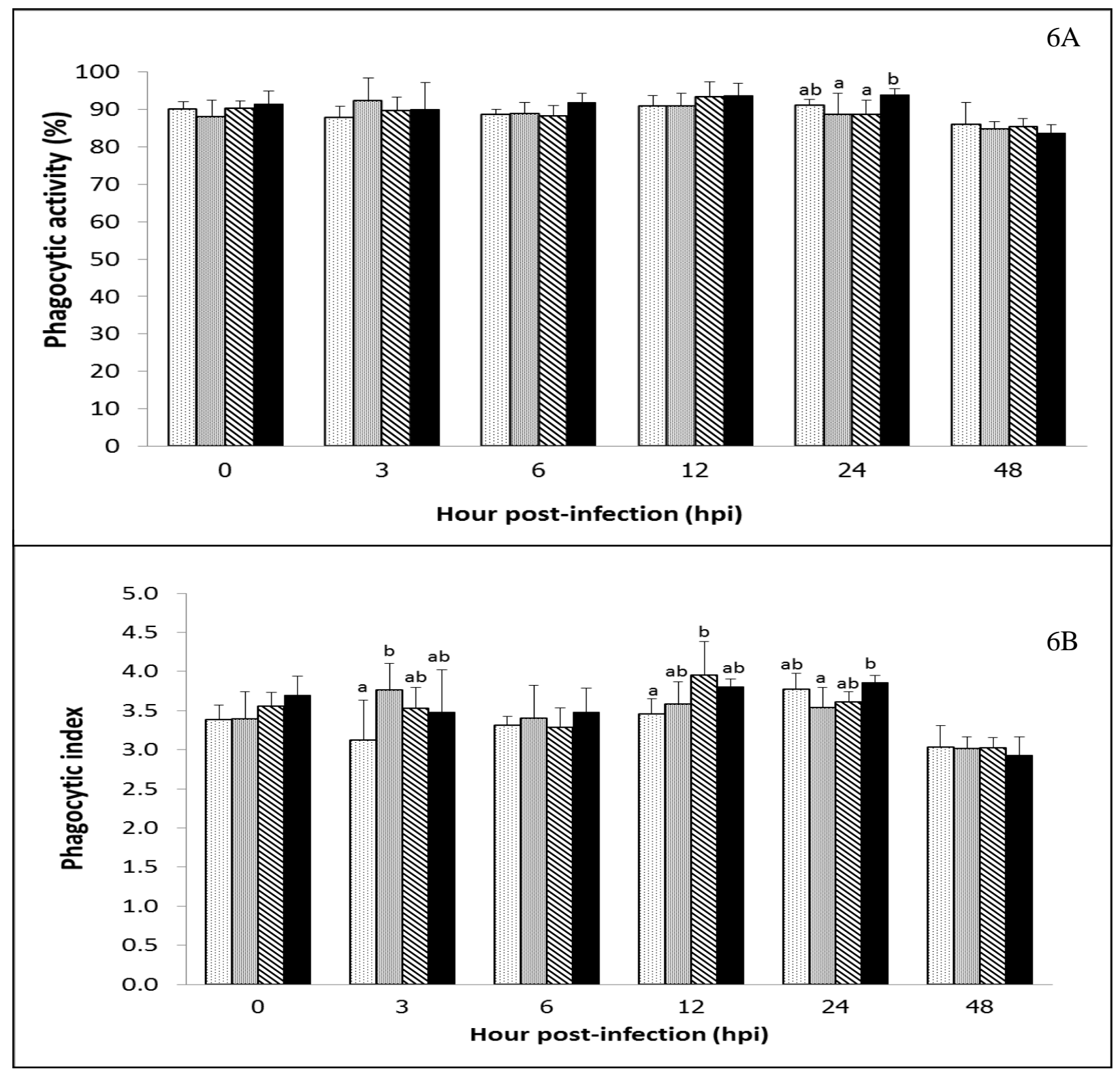

Fno-free fish (G1) Fno-free fish + S. agalactiae $(\mathrm{G} 3)$

Fno-infected fish (G2)

Fno-infected fish + S. agalactiae $($ G4) 
Figure 7
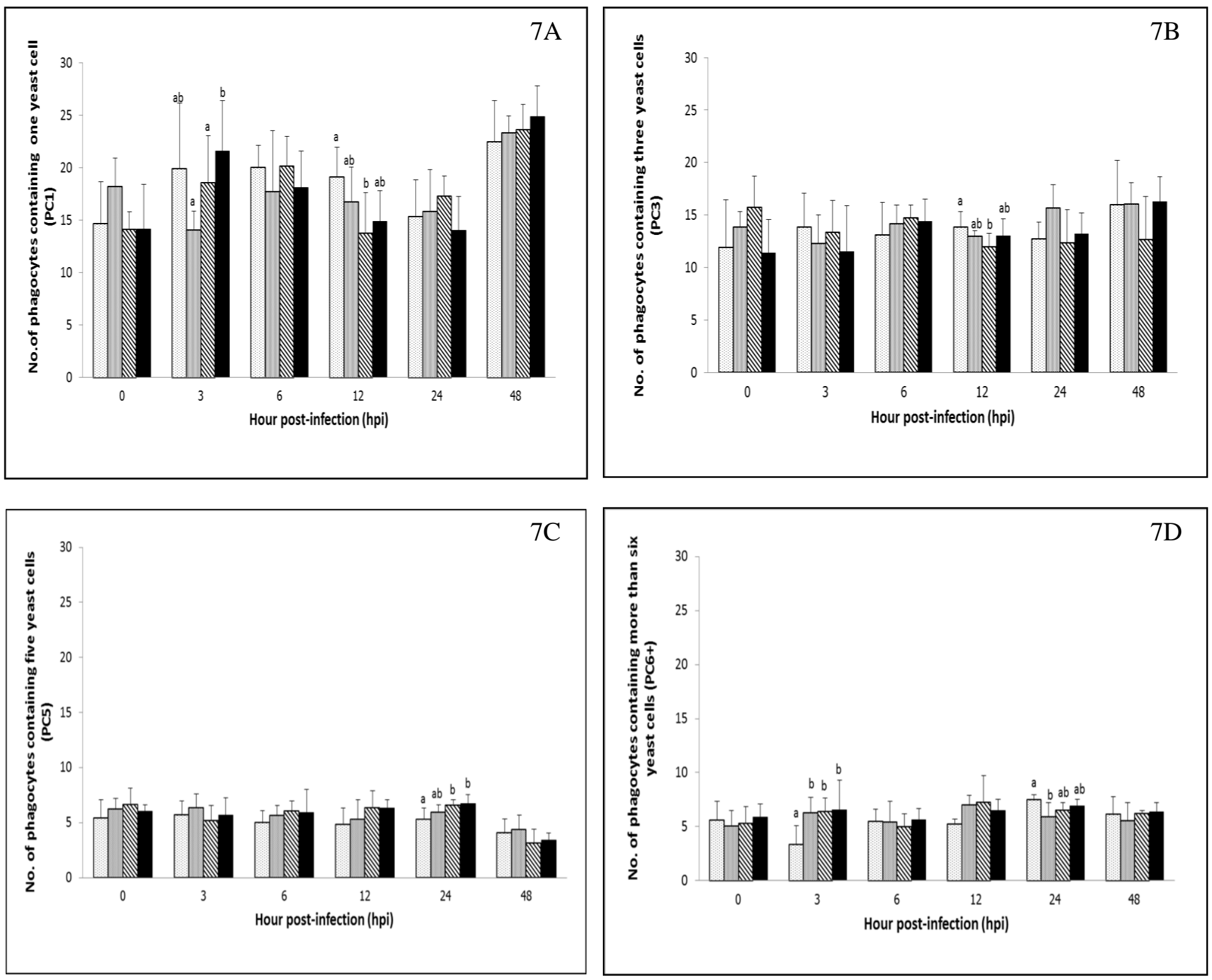

Fno-free fish (G1)

Fno-free fish + S. agalactiae (G3)

Fno-infected fish (G2)

Fno-infected fish + S. agalactiae $(\mathrm{G} 4)$ 
Figure 8
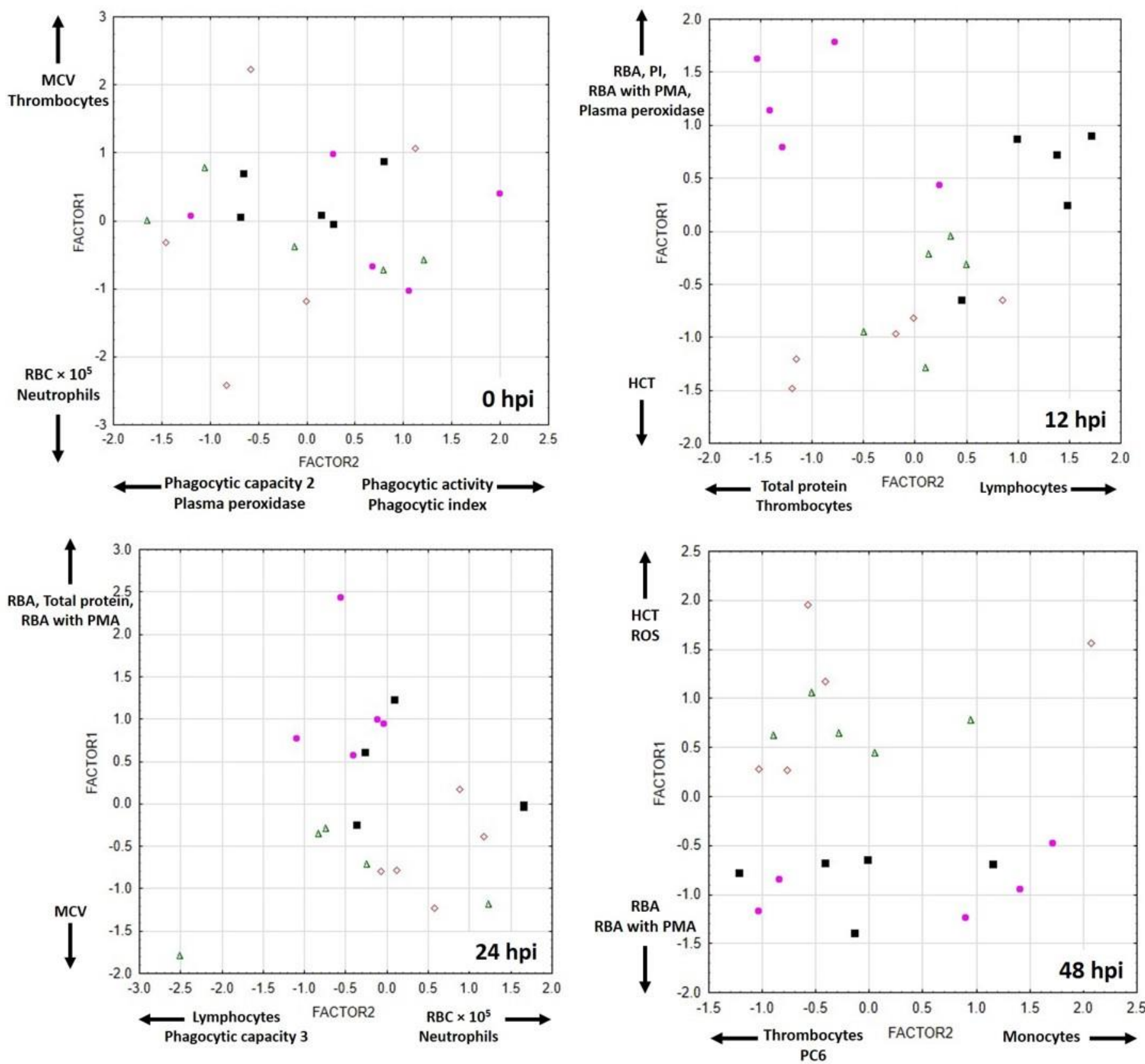

\section{$\diamond \mathrm{G} 1 \Delta \mathrm{G} 2 \square \mathrm{G} 3 \triangle \mathrm{G} 4$}

